\title{
Der kleine Unterschied
}

\section{Nicolas Diehma, Irene Pill ${ }^{b}$ und Frederic Baumann ${ }^{a}$}

a Universitätsklinik für Angiologie, Inselspital Bern

N. Diehm ist ein süddeutscher Angiologe, der sich seit 10 Jahren in der Schweiz sehr wohl fühlt.

F. Baumann ist ein in Zürich und Deutschland aufgewachsener Arzt mit schweizerischem Pass, norddeutscher Mutter und schweizerischem Vater.

b Service rund um Kultur

I. Pill ist eine süddeutsche Historikerin und Germanistin mit Auslandserfahrungen u. a. in der Schweiz, China und den USA, die heute u. a. als Dozentin für Interkulturelle Kompetenzen arbeitet.
Korrespondenz:

Prof. Dr. med. Nicolas Diehm Universitätsklinik für Angiologie Inselspital Bern

CH-3010 Bern

Tel. 0316323034

Fax 0316324793

nicolas.diehm[at]insel.ch

\section{Absicht}

Dieser Artikel möchte einen Einblick in die wichtigsten Mentalitäts- und Sprachunterschiede zwischen Schweizern und Deutschen gewähren mit dem Ziel, deren interkulturelle Begegnung zu erleichtern.

\section{Hintergrund}

Die Schweiz gilt für viele deutsche Mediziner(innen) als attraktive Arbeitsstätte. Aus verschiedenen Gründen zieht es jedes Jahr mehrere Hundert deutsche Medizinfrauen und -männer in das Land des Wilhelm Tell. Allein im Jahre 2008 waren es 842 Personen. Inzwischen bringt jede vierte in der Schweiz tätige Medizinperson ein ausländisches Arztdiplom mit und 56\% davon sind Deutsche. Am Inselspital haben aktuell 24\% aller tätigen Ärzte ein deutsches Arztdiplom und beim Pflegepersonal stammen 8\% aus dem «grossen Kanton». Somit ist die interkulturelle Kommunikation zwischen Schweizern und Deutschen ab dem ersten Arbeitstag ein relevantes Thema mit klassischen Ähnlichkeitsfallen, die im Alltag für Vertreter beider Kulturen zum Fallstrick werden können.

\section{Unterschiede in Sprache und Mentalität}

\section{Sprache}

Aus Deutschland stammende Ärzte werden, zusätzlich zur amtlichen Viersprachigkeit in der Schweiz, mit verschiedensten und zum Teil sehr unterschiedlichen Mundarten konfrontiert. Diese sind auf die geographische Zerklüftung der Schweiz und der eingeschränkten Mobilität der Schweizer bis zu Beginn des 20. Jahrhunderts zurückzuführen.

Es ist wichtig zu bedenken, dass Schweizer im Gespräch mit Deutschen vorzugsweise Hochdeutsch sprechen. Was viele Deutsche nicht wissen, ist, dass sich Hochdeutsch für Schweizer oftmals wie eine Fremdsprache anfühlt. Hingegen wissen viele Schweizer nicht, dass einige der Wörter, die für sie perfekt Hochdeutsch sind, im Umgang mit Deutschen für Verwunderung sorgen können: Zahlreiche Helvetismen wie z.B. Aufsteller (gute Nachricht), verunfallen (einen Unfall erleiden) oder allfällig (etwaig) sind Eigenheiten des schweizerischen Hochdeutsch.

Wer also glaubt, dass Schweizer und Deutsche aufgrund der Ähnlichkeit ihrer geschriebenen Muttersprache ähnlich verbal kommunizieren, irrt gewaltig. Deutsche, die nicht fliessend Schweizer- deutsch sprechen, sollten hierauf im Alltag verzichten, da germanisiertes Schweizerdeutsch in der Schweiz als Nachäffung empfunden werden kann [1].

Zudem ist zu bedenken, dass Schweizer im Gegensatz zu Deutschen oftmals die indirekte Kommunikation pflegen (Tab. 1). Der Schweizer interagiert öfter im Konjunktiv, der Deutsche hingegen fühlt sich eher im Indikativ wohl. Während der Deutsche «will», «würde» der Schweizer «gerne». Der Deutsche «muss unbedingt», während der Schweizer «eventuell sollte». Der Deutsche «kriegt» leider oftmals, der Schweizer «hätte gerne» ein Bier.

Das im schweizerischen Sprachgebrauch an viele Sätze angehängte «oder» suggeriert, dass man seine Meinung zwar äussert, diese aber nicht als absolut gilt. Der grosse Stellenwert der eidgenössischen Konsensfähigkeit der Schweizer spiegelt sich also oft in ihrer Alltagssprache wider.

\section{Mentalität}

Die Mentalität kann sich zwischen Deutschen und Schweizern deutlicher unterscheiden, als dies bei Schwaben und Berlinern der Fall sein mag. Wie sich auch die Mentalität der Einwohner Deutschlands lokoregional deutlich unterscheiden kann, trifft dies für die von einem kleinräumigen Föderalismus seit langer Zeit geprägte Schweiz zu. Die Untergliederung der Eidgenossenschaft in 26 Kantone ist also nicht nur politischer Natur, sondern immer auch Ausdruck unterschiedlicher Kulturen und Mentalitäten. Geert Hofstede unternahm einen Versuch, kulturelle Unterschiede zwischen verschiedenen Ländern zu quantifizieren [2]. Er zeigte, dass nationale Kulturgruppen einen wesentlichen Einfluss auf die Organisation und Führung von Unternehmen haben. Hierbei identifizierte er insgesamt fünf Kulturdimensionen, von denen zwei hier relevant sind:

\section{Tabelle 1}

Beispiele indirekter und direkter Kommunikation.

\section{Indirekt (schweizer- Direkt (deutsch)} deutsch)

Mer söttet no einisch Sie haben da einige grobe zämecho u das Manuskript Fehler gemacht. dürrego.

I gloube, da heimer d Möglichkeit, viel meh rungen komplett verfehlt Sie haben die Anfordeussezhole. 


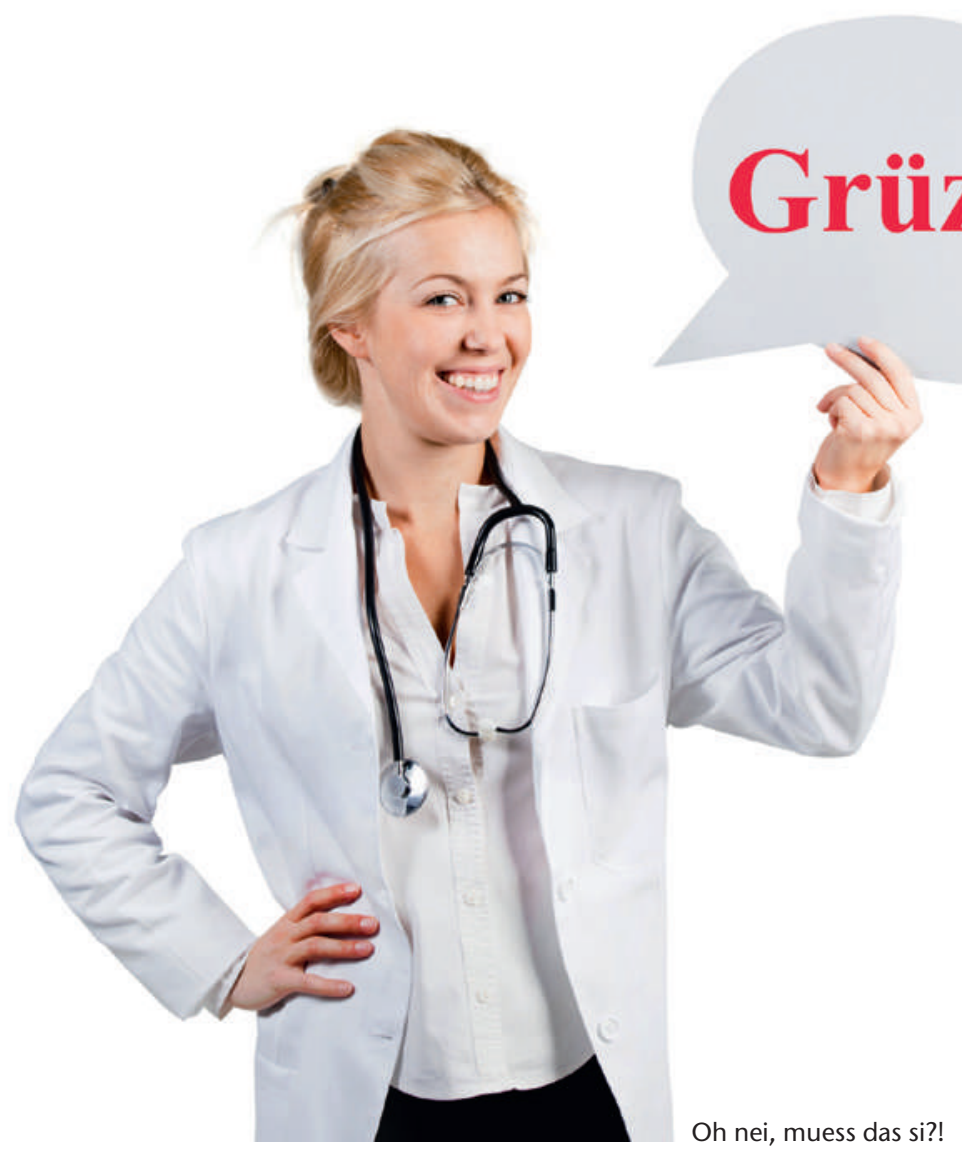

- Die Machtdistanz gibt Auskunft über den Umgang mit Macht und Ungleichheit in einem Land. Sie definiert das Ausmass, bis zu dem weniger mächtige Individuen erwarten und akzeptieren, dass Macht ungleich verteilt ist.

- Die Maskulinität versus Feminität zeigt, wie die Rollen zwischen den Geschlechtern in einer Kultur verteilt werden.

Schweizer mit geringerer Machtdistanz gehen mit sozialen Beziehungen sorgsamer um und versuchen, die Ungleichheit untereinander geringer zu halten. Sie akzeptieren hierarchische Organisationsstrukturen und Kommunikation weniger gern und beziehen Mitarbeiter mehr in Entscheidungsfindungen ein als Individuen aus Kulturen mit höherer Machtdistanz. Schweizer sind in der Regel von folgenden Eigenschaften geprägt: Harmoniebedürftigkeit, Unabhängigkeit, dem Anspruch «gleiche Rechte für alle» und einem fürsorglichen Führungsstil, der wenig Kontrolle durch die Führenden erfordert. Dies findet auch darin Ausdruck, dass das «per Du» in der Schweiz rascher eingeführt wird und zusätzlich der Titel in der Anrede rasch auf der Strecke bleibt.

Ein für Deutsche selbstverständlich selbstbewusster Auftritt und rhetorisches Geschick können von einem Schweizer als ungewohnt offensiv wahrgenommen werden. Spielerische Wortgefechte, rhetorische Schlagabtausche oder «Rechthabereien» sind der Schweizer Seele fremd. Zudem werden in Deutschland Konflikte offener ausgetragen und Aggressionen eher ausgelebt als in der Schweiz [3]. Schweizer können diese wortgewandte und direkte deutsche Art, Konflikte anzusprechen als unangemessen herrisch und arrogant empfinden.

Im Gegensatz zur deutschen Hierarchie-Gläubigkeit gilt es in der Schweizer Realität als rücksichtslos, den Diskussionspartner um jeden Preis von der eigenen Meinung überzeugen zu wollen.

Im Gegensatz zur geringeren Machtdistanz ist die Schweizer Gesellschaft maskuliner als die ihrer nördlichen Nachbarn, wobei anzumerken ist, dass sowohl die Schweizer als auch die Deutschen im internationalen Vergleich als maskulin zu bezeichnen sind. Verglichen mit einer femininen Kultur hat eine maskuline Kultur die Tendenz, Herausforderungen, Einkommen und Fortschritt als wichtig zu erachten und entsprechend durchsetzungsfähige und harte Männer zu idealisieren. In maskulinen Ländern lebt man, um zu arbeiten und arbeitet nicht, um zu leben - die Arbeit gilt als Lebenswerk.

Zusammengefasst bedeutet dies, dass Vorgesetzte in der Schweiz ihren Führungsanspruch ohne weitere Diskussion akzeptiert sehen möchten; sie vermeiden es aber zugleich tunlichst, diesen Machtanspruch gegenüber den Mitarbeitenden verbal oder symbolisch auszuleben. Eine Führungskraft muss sich durch Leistung (Maskulinität) beweisen und kann sich somit weniger auf formale oder hierarchische Macht (Machtdistanz) berufen.

\section{Kriterien erfolgreicher Kommunikation zwischen Deutschen und Schweizern}

Gemäss Lechner und Thomas [4] bedürfen folgende Schweizer Kulturstandards für Deutsche in der Schweiz besonderer Beachtung:

\section{Konsensorientierung}

Diese ist ein zentrales Element des schweizerischen politischen Systems. Dem Schweizer Bürger wird politisch eine grosse Verantwortung aufgetragen, indem er in Entscheidungsfindungen miteinbezogen wird. Die Konsensorientierung ist oft Bestandteil des schweizerischen Alltags, weshalb es einen deutschen Arzt nicht verwundern soll, wenn er sich in der Schweiz verhältnismässig oft mit «interdisziplinären» Kolloquien und Rapporten konfrontiert sieht. Zudem wird die Konsensfindung in der Schweiz als Folge der geringeren Machtdistanz viel homogener praktiziert im Sinne, dass jede(r) unabhängig von der Hierarchiestufe von seinem Mitspracherecht Gebrauch machen kann. 


\section{Gesicht wahren}

Tugenden wie Loyalität und Wertschätzungen haben in der Schweiz besondere Bedeutung. Im täglichen Umgang fällt einem aus einem deutschen Umfeld Kommenden schnell auf, dass es in der Schweiz unabdingbar ist, dem Gegenüber stets die Wahrung seines Gesichtes zu ermöglichen und den Dialog trotz möglicherweise grösstmöglichem inhaltlichem Dissens zu wahren.

\section{Etikette}

Höflichkeit wird in der Schweiz grossgeschrieben. Es mag die Geduld eines manchen Deutschen beanspruchen, wenn sich beispielsweise eine Bäckereiverkäuferin trotz langer Warteschlange (die in der Schweiz üblicherweise gesitteter ist als jene in Deutschland) für eine persönliche Betreuung der Kunden Zeit nimmt. Ob dieser langsamere, aber persönlichere Stil oder die deutsche Effizienz, die von einem Schweizer als kaltschnäuzig empfunden werden mag, für die Koronarien des Einzelnen besser ist, muss jeder für sich selbst herausfinden.

\section{Zurückhaltung/Diskretion}

Die Schweizer leben Zurückhaltung und Bescheidenheit vor. Diese Bescheidenheit der Eidgenossen äussert sich insgesamt dadurch, dass mit Leistungen nicht geprahlt wird, egal, ob sich dies auf die Motorleistung des Wagens oder auf die Anrede mit «Herr Doktor» oder «Herr Professor» bezieht. Wenngleich ein in Deutschland sozialisierter Arzt diese Zurückhaltung durchaus als Selbstverleugnung empfinden mag, so empfiehlt es sich doch, in der Schweiz eine höfliche Unterwürfigkeit zu üben. Für Deutsche in der Schweiz bedeutet dies, dass sie ihre ihnen angeborene Direktheit zu kontrollieren versuchen und sich zurücknehmen sollten.

\section{Zuständigkeitsdenken}

Ein weiteres Element, das sich aus der föderalistischen Struktur der Schweiz ableitet, ist das Zuständigkeitsdenken. So wie die einzelnen Kantone über hohe Eigenständigkeit und Selbstbestimmung verfügen, gilt in der Arbeitswelt das Verständnis, dass jeder für seinen klar abgegrenzten Aufgabenbereich zuständig ist, in den sich niemand einmischt. Dennoch hält man sich an das übergeordnete Ziel, welches das Wohlergehen des gesamten Unternehmens in den Vordergrund stellt.

\section{Patriotismus}

Im Gegensatz zum deutschen Kulturraum, in dem Patriotismus insbesondere unter jungen Leuten heute relativ schwach ausgeprägt ist, ist die Liebe zum Vater- land und dessen Traditionen in der Schweiz weitverbreitet. So vermarktet man heute in der Schweiz die «Swissness» [5]; deren positiv konnotierte Attribute, die Fairness, Präzision, Zuverlässigkeit, politische Stabilität, Natürlichkeit, Genauigkeit und Sauberkeit, sind heute deutlich aggressiver, als dies in Deutschland für deutsche Attribute der Fall ist.

Schweizer identifizieren sich sehr mit ihrem Kanton und ihrem Land und erwarten von Ausländern Anerkennung und Respekt für die Schweiz. Die Abgrenzung gegenüber Deutschland hat in der Schweiz einen hohen Stellenwert. Entsprechend besteht bei Schweizern das Bedürfnis nach Abgrenzung gegenüber dem «grossen Kanton», während die Deutschen im Allgemeinen oberflächlich betrachtet in der Schweiz ein idealisiertes Deutschland sehen und ihr sehr wohlwollend gegenüberstehen [6].

\section{Zusammenfassung}

Zusammengefasst sollte man sich als Deutscher, der in der Schweiz primär nicht als «Sauschwabe» [7] auffallen möchte, an eine Empfehlung der Landeszentrale für politische Bildung in Baden-Württemberg halten und «nicht zu laut und nicht zu schnell reden» und agieren und «im Ausdruck jegliche Vehemenz vermeiden» $[1]$.

So unterschiedlich beide Kulturen auch sein mögen - verbindende Gemeinsamkeiten unter Kollegen sind immer zu finden. Sollte jedoch der Ernstfall des interkulturellen Kommunikations-GAUs zwischen Schweizern und Deutschen eintreten, steht zuletzt die Hoffnung auf beiderseitige kulturelle Akzeptanz, Toleranz, Geduld und Gutmütigkeit, die dieser Artikel auch ein Stück weit vermitteln soll.

\section{Literatur}

1 Sitzler S. Grüezi und Willkommen. Die Schweiz für Deutsche. Bonn: Landeszentrale für politische Bildung Baden-Württemberg;2009.

2 Hofstede G. Cultures and Organizations. Software for the Mind. 2010

3 Unter Leidgenossen. Süddeutsche Zeitung vom 5./6. Februar 2011. S. V2/15

4 Lechner T, Thomas A. Kulturstandards in der Schweiz. Trainingsprogramm für Manager, Fach- und Führungskräfte. Göttingen: Vandenhoeck \& Ruprecht; 2011.

5 http://de.wikipedia.org/wiki/Swissness, Seite geöffnet am 26.11.2012.

6 Berg A. Deutsch-schweizerisches Verhältnis Nicht ohne meinen Bruder. Die Zeit vom 1.7.2010.

7 Widmer U. Kuhschweizer und Sauschwabe. Frankfurter Allgemeine Zeitung vom 31.3.2009. 